\title{
A Standardized Method Based on Pigmented Epidermal Models Evaluates Sensitivity Against UV-Irradiation
}

\author{
Freia F. Schmid \#1, Florian Groeber-Becker ${ }^{\# 1}$, Stefanie Schwab ${ }^{2}$, Sibylle Thude 2, Matthias Goebeler ${ }^{3}$, \\ Heike Walles ${ }^{1,4}$ and Jan Hansmann 1,4 \\ ${ }^{1}$ Translational Center Regenerative Therapies, Fraunhofer Institute for Silicate Research, Würzburg, Germany; ${ }^{2}$ Department of Cell and Tissue \\ Engineering, Fraunhofer Institute for Interfacial Engineering and Biotechnology, Stuttgart, Germany; ${ }^{3}$ Department of Dermatology, Venereology and \\ Allergology, University Hospital Würzburg, Würzburg, Germany; ${ }^{4}$ Department of Tissue Engineering \& Regenerative Medicine, University Hospital \\ Würzburg, Würzburg, Germany
}

\begin{abstract}
To protect the human skin from extensive solar radiation, melanocytes produce melanin and disperse it via melanosomes to keratinocytes in the basal and suprabasal layers of the human epidermis. Moreover, melanocytes are associated with pathological skin conditions such as vitiligo and psoriasis. Thus, an in vitro skin model that comprises a defined cutaneous pigmentation system is highly relevant in cosmetic, pharmaceutical and medical research. Here, we describe how the epidermal melanin unit can be established in vitro. Primary human melanocytes were introduced into an open source reconstructed epidermis. Following 14 days at the air liquid interface, a differentiated epidermis had formed and the melanocytes were located in the basal layer. The functionality of the epidermal melanin unit could be shown by the transfer of melanin to the surrounding keratinocytes, and a significantly increased melanin content of models stimulated with either UV-radiation or the melanin precursor dihydroxyphenylalanine (DOPA). An $U V_{50}$ assay was developed to test the protective effect of melanin. In analogy to the $\mathrm{IC}_{50}$ value in risk assessment, the $U_{50}$ value facilitates a quantitative investigation of harmful effects of natural UV-radiation to the skin in vitro. Employing this test, we could demonstrate that the melanin content correlates with the resilience against simulated sunlight, which comprises 2.5\% UVB and 97.5\% UVA. Besides demonstrating the protective effect of melanin in vitro, the assay was used to determine the protective effect of a consumer product in a highly standardized setup.
\end{abstract}

\section{Introduction}

Besides guarding the inner organs from mechanic, chemical or thermal injury, the skin is the first line of protection against extensive solar radiation (Alonso and Fuchs, 2003; Brenner and Hearing, 2008). The sun emits light of various wavelengths, whereby the non-visible ultraviolet (UV) spectrum has the greatest potential to elicit tissue damage (Ullrich, 2002). While UVBlight can only penetrate into the upper layers of the epidermis, it is capable of directly inducing mutations. In contrast, UVA-light reaches into deeper skin layers, where it generates free radicals such as reactive oxygen species. Although the formation of reactive oxygen species is an ubiquitous process, extensive UV-radiation can overwhelm the antioxidant defense system and lead to oxidative stress in the skin (Rittié and Fisher, 2002). Thereby, reversible and irreversible subcellular damage to nucleic acids, proteins, free amino acids and proteins of the connective tissue is caused (Kadekaro et al., 2003). In its mildest form, such damage entails sunburn features in the skin. However, when UV-radiation doses accumulate, the susceptibility to different forms of skin cancer is increased (Ullrich, 2002).

To prevent extensive UV-radiation-induced damage, the skin has developed a specialized system that protects cells in the epidermis. During embryogenesis melanoblasts migrate from the neural crest to the skin, differentiate to melanocytes and form dendritic extensions to the surrounding keratinocytes (Mayer, 1973; Wang et al., 2016). This anatomical connection was defined by Fitzpatrick and Breathnach as the "epidermal

\footnotetext{
\# contributed equally

Received December 21, 2017; Accepted April 11, 2018; Epub April 13, 2018; @ The Authors, 2018. ALTEX 35(3), 390-396. doi:10.14573/altex.1712211

Correspondence: Florian Groeber-Becker, PhD, Fraunhofer-Institut für Silicatforschung ISC, Translationszentrum Regenerative Therapien, Röntgenring 11, 97070 Würzburg, Germany

(florian.kai.groeber-becker@isc.fraunhofer.de)
}

This is an Open Access article distributed under the terms of the Creative Commons Attribution 4.0 International license (http://creativecommons.org/licenses/by/4.0/) which permits unrestricted use, distribution and reproduction in any medium, provided the original work is appropriately cited. 
melanin unit". It has been estimated that one melanocyte is in contact with 36 keratinocytes of the basal and suprabasal layer (Fitzpatrick and Breathnach, 1963; Nordlund, 2007). In melanocytes, the pigment melanin is produced and dispersed to the keratinocytes via melanosomes. Upon reaching the keratinocytes, melanin granules accumulate above the cell nuclei to protect the deoxyribonucleic acid (DNA) from UV-radiation-induced damage such as pyrimidine dimer formation. Thereby, the "epidermal melanin unit" is also responsible for the color of the skin, which is called constitutive pigmentation in its basal state and facultative pigmentation after responding to environmental stimuli such as sunlight. In addition to the direct safeguard capacity of adsorbing and scattering UV-radiation, melanin protects the skin by photo-oxidizing and scavenging free radicals (Brenner and Hearing, 2008).

In vitro, the epidermal melanin unit could be established in tissue-engineered, reconstructed human epidermis. In addition to human keratinocytes, these models also contain melanocytes (Liu et al., 2007). Such so-called pigmented epidermal models resemble the anatomical structure of human epidermis including the interaction between human keratinocytes and melanocytes that ultimately results in macroscopically visible tanning (Bessou et al., 1995). Hence, pigmented reconstructed human epidermis may be employed as an alternative to animal testing in cosmetic, pharmaceutical and medical research, e.g., for sun protection, self-tanning agents or skin whitener. However, no study has shown a direct quantitative correlation between melanin content and the capacity to withstand natural UV-radiation in vitro, yet.

The study presented here was initiated to assess the influence of the melanin content in pigmented reconstructed human epidermis on the capacity of the tissue to resist UV-irradiation. Therefore, a pigmented skin model based on the open source reconstructed human epidermis (OS-REp) (Groeber et al., 2016b; Mewes et al., 2016) was generated. Following, the effect of UV-irradiation, different cell donors and the melanin precursor dihydroxyphenylalanine (DOPA) were correlated to melanin synthesis. Moreover, a test procedure that quantifies the resilience of a tissue to solar radiation was derived. Finally, the protective effect of a commercially available sun protection agent was demonstrated with the novel assay. To reflect a realistic UV-irradiation, a mixture of UVA- and UVB-radiation was used according to the global solar reference spectrum.

\section{Material and methods}

\section{Cell isolation}

Human epidermal keratinocytes were isolated from foreskin biopsies of 2- to 5-year-old donors with approval of the local ethics committee (approval number IGBZSF-2012-078) after confirmed consent of their guardians. The cell isolation procedure was based on a previously published protocol (Groeber et al., 2016a). Briefly, biopsies were washed, minced and digested with dispase (Life Technologies, Darmstadt, Germany) to dissociate the epidermis from the dermis. Thereafter, the epidermis was trypsinized (Life Technologies) to generate single-cell suspensions. Keratinocytes were cultured in EpiLife ${ }^{\circledR}$ medium supplemented with $0.2 \% \mathrm{v} / \mathrm{v}$ bovine pituitary extract, $1 \mu \mathrm{g} / \mathrm{ml}$ recombinant human insulin-like growth factor- $1,0.18 \mu \mathrm{g} / \mathrm{ml}$ hydrocortisone, $5 \mu \mathrm{g} / \mathrm{ml}$ bovine transferrin and $0.2 \mathrm{ng} / \mathrm{ml}$ human epidermal growth factor (all from Life Technologies). Additionally, human melanocytes were obtained from the epidermis of adult skin biopsies of 19- to 61-year-old donors and were isolated from the epidermal part by the same procedure as described for the keratinocytes. The skin phototype of all donors was between 2 and 4 . In contrast to the isolation of keratinocytes, cells were seeded and cultured in Melanocyte Growth Medium ready (PromoCell, Heidelberg, Germany).

\section{Generation of epidermal models}

Skin models were generated on the polycarbonate membrane of respective cell culture inserts (diameter $0.47 \mathrm{~cm}^{2}$, pore size $0.4 \mu \mathrm{m}$; Nunc ${ }^{\mathrm{TM}}$, Waltham, USA). To generate melanocyte-free epidermal models, keratinocytes were seeded at a cell density of $5 \times 10^{5}$ cells $/ \mathrm{cm}^{2}$ in $200 \mu \mathrm{l}$ EpiLife ${ }^{\circledR}$ medium supplemented with $0.2 \% \mathrm{v} / \mathrm{v}$ bovine pituitary extract, $1 \mu \mathrm{g} / \mathrm{ml}$ recombinant human insulin-like growth factor- $1,0.18 \mu \mathrm{g} / \mathrm{ml}$ hydrocortisone, $5 \mu \mathrm{g} / \mathrm{ml}$ bovine transferrin, $0.2 \mathrm{ng} / \mathrm{ml}$ human epidermal growth factor (all from Life Technologies) and $1.5 \mathrm{mM} \mathrm{CaCl}_{2}$ (Sigma-Aldrich, Steinheim, Germany). To ensure sufficient nutrient supply, inserts were cultured in $2 \mathrm{ml}$ medium in 6-well-plates. Medium was changed after $24 \mathrm{~h}$ to EpiLife ${ }^{\circledR}$ air-liquid-interface medium that additionally contains $73 \mu \mathrm{g} / \mathrm{ml} \mathrm{L}$-ascorbic acid 2-phosphate (Sigma-Aldrich). During culture, medium was replaced by fresh air-liquid-interface medium three times per week and models were cultured at $37^{\circ} \mathrm{C}$ and $5 \% \mathrm{CO}_{2}$ in a humidified incubator. For the generation of pigmented skin models, melanocytes were mingled with keratinocytes at a ratio of 1:6 applying the same culture conditions as described for the melanocyte-free tissues.

\section{Histological analysis}

Samples were fixed in Roti ${ }^{\circledR}$ Histofix (Roth, Karlsruhe, Germany) for $1 \mathrm{~h}$, washed in tap water for $2 \mathrm{~h}$ and embedded in paraffin. Subsequently, histological cross-sections of $3 \mu \mathrm{m}$ were obtained. Prior to the staining, slides were deparaffinized and rehydrated. For the identification of melanin, a Fontana-Masson stain was conducted, whereby melanin is visualized by staining with silver nitrate. Counterstaining was done with nuclear fast red aluminium sulphate solution. Immunolabelling for Melan-A was performed to identify melanocytes while melanosomes were immunostained using the HMB45 antibody. Samples were exposed to citrate buffer to allow demasking of epitopes and were permeabilized with 1\% Triton X-100 (Sigma-Aldrich) in Dulbecco's phosphate buffered saline (DPBS) (Sigma-Aldrich) for $10 \mathrm{~min}$. Slides were then incubated with Melan-A or HMB45 antibodies (both obtained from Dako, Hamburg, Germany) at a dilution of $1: 50$ at $4{ }^{\circ} \mathrm{C}$ overnight, washed and subsequently stained with appropriate secondary antibodies for a further $30 \mathrm{~min}$. Subsequently, slides were washed again with DPBS and cell nuclei counterstained with 4',6-diamidino-2-phenylindole (DAPI) at a dilution of 1:1000 (Serva Electrophoresis, Heidelberg, Germany). 


\section{Melanin quantification}

Skin models were digested with dispase (Life Technologies) to dissociate skin equivalent from the membrane. Epidermal layers were removed from the cell culture insert and transferred to a

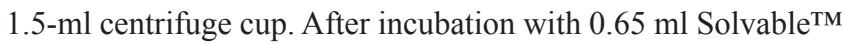
(Perkin Elmer, Rodgau, Germany) for $30 \mathrm{~min}$ at $60^{\circ} \mathrm{C}$, melanin was quantified by measuring the absorbance at $405 \mathrm{~nm}$ with a spectrophotometer (Infinite 200M; Tecan, Maennerdorf, Switzerland). A serial dilution of synthetic melanin at a range of 0 to $50 \mu \mathrm{g} / \mathrm{ml}$ allowed quantification of the melanin concentrations in the skin samples.

\section{$U V$-irradiation}

UV-irradiation of skin models was performed with a BIOSUN system (Vilber Lourmat, Eberhardzell, Germany). The BIO-SUN irradiation system has integrated UV sensors and a microprocessor. The emission of the UV light is continuously monitored and the irradiation stops automatically when the dose matches the desired set point. After the transfer of skin models to a 6-well plate without medium, samples were irradiated with UVA or UVB at different doses. Each model was irradiated three times with an interval of $24 \mathrm{~h}$ between treatments. Following post-incubation of $48 \mathrm{~h}$ after the last irradiation, the melanin content was quantified. For post-incubation, tissue models were transferred to air-liquid-interface medium.

\section{UV 50 assay}

In order to obtain information on the sensitivity of the model towards solar radiation, a $\mathrm{UV}_{50}$ assay was established. The tis- sues were irradiated with increasing doses of simulated sunlight $\left(0-18 \mathrm{~J} / \mathrm{cm}^{2}\right)$. To mimic a realistic spectrum, the light comprised $97.07 \%$ UVA and $2.93 \%$ UVB. Subsequently, tissue viability was assessed using a 3-(4,5-dimethylthiazol-2-yl)-2,5-diphenyl tetrazolium bromide (MTT) assay 3 days after the irradiation. $\mathrm{UV}_{50}$ was calculated as the dose at which tissue viability had decreased to $50 \%$ of the initial viability by plotting percent viability (linear y axis) against the UV dose. $U_{50}$ values were determined by a sigmoid dose-response curve showing the characteristic dose-dependent decrease of viability. By interpolation, the dose at which the percent viability had dropped to $50 \%$ was considered the $\mathrm{UV}_{50}$ value.

\section{DOPA treatment}

$25 \mu 1$ DOPA was applied topically at various concentrations $(0$, $0.3,1$ and $3 \mathrm{mg} / \mathrm{ml}$ in DPBS) starting at day 10 of the air-liquid-interface phase three times every $24 \mathrm{~h}$. After post-incubation for another $48 \mathrm{~h}$, the melanin content was quantified.

\section{Characterization of sun protection agent}

To assess the effect of a commercially available sun lotion, skin models were treated topically with $25 \mu 1$ of the product (Sun Dance Ultra-sensitive SPF 30, m-drogerie markt GmbH \& Co. $\mathrm{KG}$; Karlsruhe, Germany). The sun lotion was distributed evenly over the surface using sterile cotton swabs. Following $30 \mathrm{~min}$ incubation at room temperature, tissues underwent a $\mathrm{UV}_{50}$ assay as described above. Untreated models served as control. Moreover, models that were not irradiated and models that were challenged with doses of artificial sunlight of 10,20 and $30 \mathrm{~J} / \mathrm{cm}^{2}$ were subjected to histological analysis.

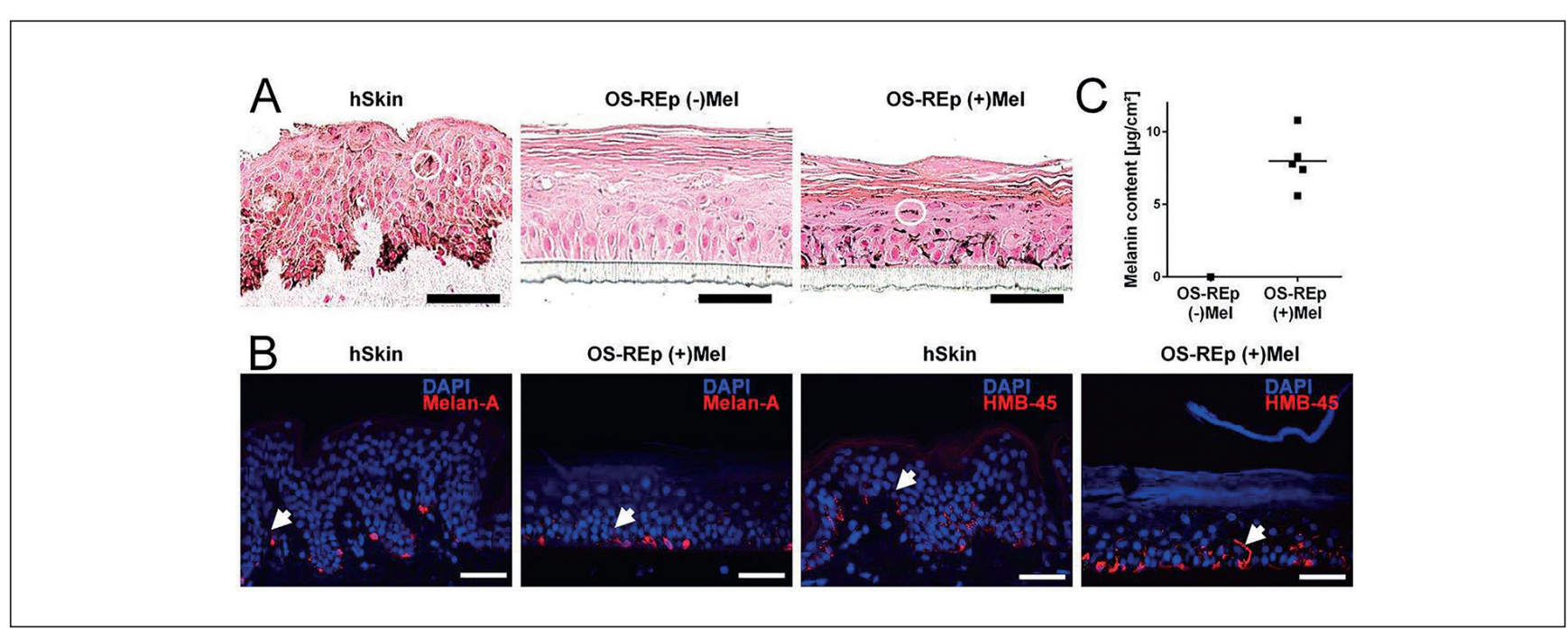

\section{Fig. 1: Histological analysis of pigmented reconstructed human epidermis}

(A) Fontana-Masson staining of non-pigmented (OS-REp (-) Mel) and pigmented epidermal models (OS-REp (+) Mel) and human skin (hSkin). The pigmented and non-pigmented epidermal models both show histological features resembling human skin. Melanocytes are located in the stratum basale and melanin granules above the cell nuclei are visible in the stratum spinosum and stratum granulosum in hSkin and OS-Rep (+) Mel (marked with a white circle). (B) Immunofluorescence staining for Melan-A and HMB-45 of OS-REp (+) Mel and human skin (hSkin). DAPI was used for counterstaining of cell nuclei. Dendritic cellular protrusions into suprabasal epidermal layers are highlighted with an arrow. (C) Melanin content of pigmented and non-pigmented epidermal models. To determine the melanin content a spectrometric melanin quantification was performed for pigmented OS-Rep generated from cells derived from five different donors. Data is shown as dot plot in which each data point represents the melanin content of one donor. Scale bar: $50 \mu \mathrm{m}$. 
Statistical analysis

Statistical analysis was performed using one-way analysis of variance (ANOVA) with Fisher LSD test using the appropriate control in each experiment as a reference. Values of $p \leq 0.05$ were considered significant.

\section{Results}

\subsection{Pigmented reconstructed}

\section{human epidermis mimics the epidermal} melanin unit of human skin

Following culture at the air-liquid-interface for 14 days, the OS-REp models highly resembled the histological architecture of human epidermis (Fig. 1). The models showed a physiological differentiation pattern with a prismatic basal layer, two to three layers of stratum spinosum with flattened keratinocytes and two to three layers of stratum granulosum with keratin granules. Moreover, differentiation was reflected by a thick corneous layer composed of cell-nucleus-free corneocytes. When melanocytes were seeded together with keratinocytes, Fontana-Masson staining revealed the localization of the former in the stratum basale.
This finding could also be confirmed by immunofluorescence staining with HMB-45 and Melan-A antibodies (Fig. 1A,B). Additionally, Fontana-Masson staining and immunolabeling allowed visualization of dendritic melanocyte protrusions into suprabasal epidermal layers. Melanin granules were also visible above the cell nuclei in cells of the stratum spinosum and stratum granulosum that did not have direct contact with melanocytes.

Spectrometric melanin quantification revealed an increased and donor-dependent melanin content in pigmented epidermal models in comparison to melanocyte-free models (Fig. 1C). The degree of pigmentation varied between 5.6 and $10.8 \mu \mathrm{g} / \mathrm{cm}^{2}$ when using melanocyte populations from different donors.

\subsection{Melanin content in pigmented epidermal models increases upon UV-irradiation or exposure to the melanin precursor DOPA}

To test if the pigmented reconstructed human epidermis responds to increasing doses of UV-irradiation, models were irradiated with 2 or $5 \mathrm{~J} / \mathrm{cm}^{2} \mathrm{UVA}$ and 20 or $40 \mathrm{~J} / \mathrm{cm}^{2} \mathrm{UVB}$ (Fig. 2A). Both UVA-irradiation doses of 2 and $5 \mathrm{~J} / \mathrm{cm}^{2}$ and the higher UVB dose of $40 \mathrm{~J} / \mathrm{cm}^{2}$ resulted in a statistically significant increase of the melanin content of $69 \%$ and $39 \%$ compared to the non-irra-

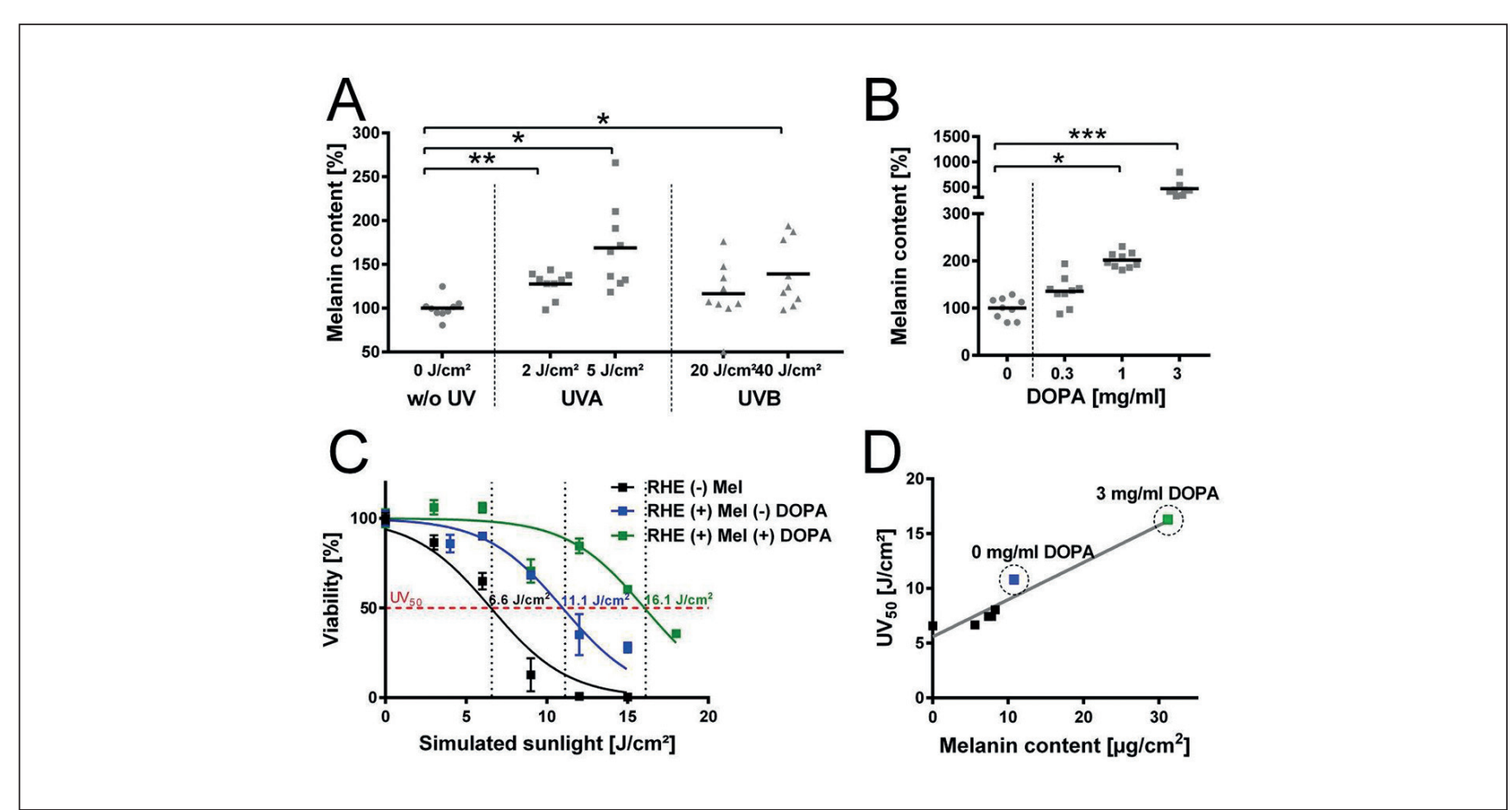

Fig. 2: Influence of UV-radiation and DOPA application on the pigmentation of epidermal models

(A) Determination of melanin content after UV-irradiation. Pigmented models were irradiated with 2 or $5 \mathrm{~J} / \mathrm{cm}^{2} \mathrm{UVA}$, or $20 \mathrm{or} 40 \mathrm{~J} / \mathrm{cm}^{2}$ UVB. (B) Melanin content after topical treatment of OS-REp (+) Mel with 0, 0.3, 1 and $3 \mathrm{mg} / \mathrm{ml}$ DOPA in DPBS. (C) Sensitivity of epidermal models to irradiation with simulated sunlight comprised of $97.07 \%$ UVA and $2.93 \%$ of UVB. Viability after irradiation with increasing doses of simulated sunlight (0-18 J/cm²) was assessed by MTT assay. Models without melanocytes (OS-REp (-) Mel), with melanocytes (OS-REp (+) Mel (-) DOPA) and after stimulation with $3 \mathrm{mg} / \mathrm{ml}$ DOPA (OS-REp (+) Mel (+) DOPA) were tested. The UV 50 value was determined by a sigmoidal dose-response curve. (D) The correlation between the pigmentation level and the $\mathrm{UV}_{50}$ value was determined. The Pearson correlation coefficient was computed and showed a strong correlation $(r=0.96)$. Data is shown as dot plots with mean value in $(A)$ and $(B)$. In (C), plots represent mean value \pm standard deviation. Statistically relevant differences are indicated by stars $\left({ }^{*} p \leq 0.05\right.$, ${ }^{* *} p \leq 0.01,{ }^{* *} p \leq 0.001, n=3$ independent experiments). 
diated controls. Moreover, exposure to DOPA dose-dependently led to an increased melanin content of the epidermal models as well (Fig. 2B).

\subsection{Melanin content correlates with UV-radiation and protective capacity}

The primary purpose of melanin is to protect skin from extensive sunlight. To investigate the sensitivity of reconstructed human epidermis against different doses of sunlight exposure, a novel test procedure to assess responses to solar irradiation was developed (Fig. 2C). To ensure realistic test conditions, we used a defined mixture of UVA (97.07\%) and UVB $(2.93 \%)$ light according to the global sunlight reference spectrum. The irradiation dose that led to a reduction of the viability of the models to $50 \%$ of the initial viability $\left(\mathrm{UV}_{50}\right)$ proved to be an appropriate parameter for this purpose. Employing this new test method, we could show a positive dose-response relationship between the melanin content of the epidermal models and the $\mathrm{UV}_{50}$ value. Melanocyte-free models revealed a $U_{50}$ value of $6.6 \mathrm{~J} / \mathrm{cm}^{2}$, and thus a higher sensitivity against UV-irradiation compared to pigmented epidermal models. Here, the presence of melanocytes, and thus melanin, in the pigmented models increased the $\mathrm{UV}_{50}$ to $11.1 \mathrm{~J} / \mathrm{cm}^{2}$. Viability could furthermore be improved by exposure to DOPA, which raised the $\mathrm{UV}_{50}$ to $16.1 \mathrm{~J} / \mathrm{cm}^{2}$, respectively. The Pearson correlation coefficient $(r=0.96)$ revealed a strong positive correlation between both parameters (Fig. 2D).

\subsection{The $U V_{50}$ assay quantifies the protective effect of a sun protection consumer product}

Epidermal models were challenged with increasing doses of artificial sunlight both with and without prior topical application of a commercially available sun lotion. In the histological analysis, we could see the occurrence of strong UV-induced hydropic degeneration at a dose of $10 \mathrm{~J} / \mathrm{cm}^{2}$ when no sun protection factor was applied (Fig. 3A). These features were more pronounced at $20 \mathrm{~J} / \mathrm{cm}^{2}$ but seemed to be diminished at an even higher dose of $30 \mathrm{~J} / \mathrm{cm}^{2}$. At this dose, cell nuclei appeared to be fragmented. If the sun lotion was applied before the experiments, no effects could be observed at $10 \mathrm{~J} / \mathrm{cm}^{2}$. A dose of $20 \mathrm{~J} / \mathrm{cm}^{2}$ only led to histological changes within the stratum corneum, whereas a high dose of $30 \mathrm{~J} / \mathrm{cm}^{2}$ resulted in visible changes in the viable cell layers (Fig. 3A). These histological findings correlated with the measured viabilities of the tissue models (Fig. 3B). Without the sun protection factor, viability was strongly decreased for low doses of artificial sunlight and led to a $U_{50}$ value of $6.7 \mathrm{~J} / \mathrm{cm}^{2}$. With the sun lotion, tissues remain viable upon treatment with higher doses and the $\mathrm{UV}_{50}$ value increased to $21.6 \mathrm{~J} / \mathrm{cm}^{2}$.

\section{Discussion}

The cutaneous pigmentation system has remarkable capacities to protect human skin from solar radiation by quenching energy from photons or scavenging reactive oxygen species. Melano-

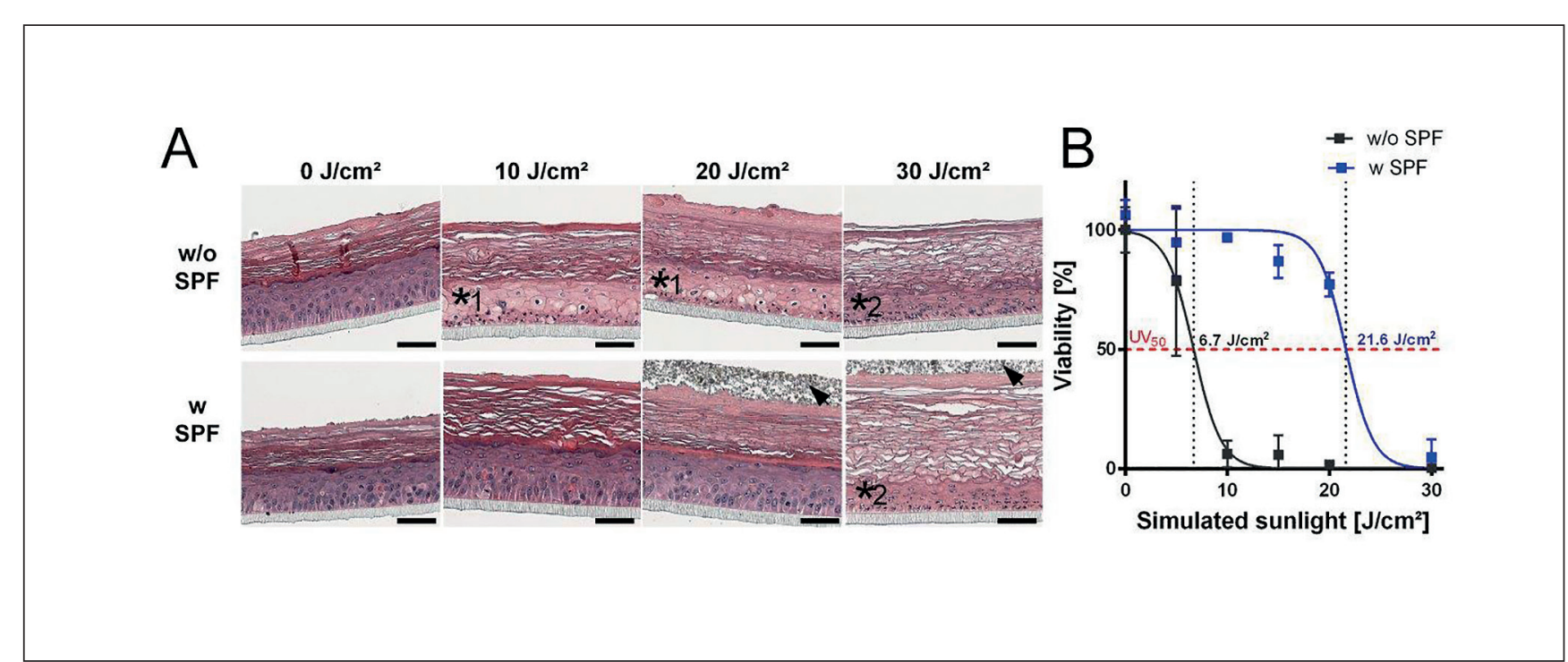

Fig. 3: Influence of sun protection factor (SPF) and UV-irradiation

(A) Histological analysis of reconstructed human epidermis after irradiation with different doses of simulated sunlight $(0,10,20$ and $30 \mathrm{~J} / \mathrm{cm}^{2}$ ) with or without SPF treatment. UV-induced damage ( ${ }^{*} 1$ : UV-induced hydropic degeneration; *2 fragmented nuclei) of the viable cell layers was visible without SPF already at a dose of $10 \mathrm{~J} / \mathrm{cm}^{2}$ simulated sunlight, whereas with SPF, damage was first visible at a dose of $30 \mathrm{~J} / \mathrm{cm}^{2}$. Scale bar: $50 \mu \mathrm{m}$. (B) Sensitivity of epidermal models against irradiation with simulated sunlight with or without SPF. Mean value and standard deviation of tissue viability $(n=3)$ after irradiation with increasing doses of simulated sunlight $\left(0-30 \mathrm{~J} / \mathrm{cm}^{2}\right)$ without SPF (w/o SPF), and with SPF (w SPF). To quantify sensitivity, $\mathrm{UV}_{50}$ values were determined by a sigmoid dose-response curve. Models without SPF showed a $U V_{50}$ value of $6.7 \mathrm{~J} / \mathrm{cm}^{2}$, whereas with SPF this value was increased to $21.6 \mathrm{~J} / \mathrm{cm}^{2}$. 
cytes are involved in different skin diseases such as vitiligo or, as indicated in recent research, also psoriasis (Arakawa et al., 2015; Wagner et al., 2015). Skin pigmentation is of high social importance since, depending on the respective cultural background, the pigmentation is desired to be either increased or decreased (Leary and Jones, 1993). Thus, there is an increasing interest to study these processes in a standardized model that mimics the in vivo situation as closely as possible.

To generate such a model, we seeded primary human melanocytes together with primary human keratinocytes at a ratio of 1:6 on polycarbonate cell culture inserts. Following the culture at the air-liquid interface, the keratinocytes formed a well-differentiated epidermis. In this environment, melanocytes remained in the basal layer of the developed epidermis and established dendritic protrusions reaching to keratinocytes both in basal and suprabasal layers. Comparable to the in vivo situation and other in vitro models, these melanosomes are arranged as a protective cap over cell nuclei to prevent damage to genetic information (Kaidbey et al., 1979; Liu et al., 2007). Since melanin is detectable within the cytoplasm of keratinocytes, a physiological transfer of melanosomes to keratinocytes, and thus a functional epidermal melanin unit, can be assumed in the model.

The pigmentation system in the epidermal models showed a considerable dynamic and responded with increased melanin production upon UVA and UVB irradiation or upon exposure to DOPA. Both reactions have been well-studied in vivo and could be demonstrated in in vitro models, where comparable effects can be observed (Wolber et al., 2008; Slominski et al., 1988; Duval et al., 2001; Yoon et al., 2003; Bessou et al., 1995). In contrast to previously published models, our model was developed following an open source policy that was previously employed to generate a non-pigmented epidermal model for risk assessment (Groeber et al., 2016b; Mewes et al., 2016). Comparable to the open source concept in the information technology field, all procedures to generate the model are freely published and thus allow a broad scientific community to contribute to the further refinement of the model (Bagozzi and Dholakia, 2006; Hertel et al., 2003; Lakhani and von Hippel, 2003).

In addition, we developed a test to quantify the resilience towards solar radiation to test whether the melanin produced in the skin models is also able to protect the skin in vitro. We irradiated tissues with increasing doses of artificial sunlight and derived a robust parameter, the $\mathrm{UV}_{50}$ value, i.e., the value that resulted in a decrease of tissue viability to $50 \%$ that corresponds to the well-known $\mathrm{IC}_{50}$ value in risk assessment. To consider a realistic UV light exposure, a defined mixture of UVA and UVB according to the global reference spectrum of sunlight was used. Without melanocytes, the $\mathrm{UV}_{50}$ value was only $6.6 \mathrm{~J} / \mathrm{cm}^{2}$ but increased depending on the respective melanin concentration up to 1.7-fold. The strong correlation between phototype and protection from UV-irradiation has already been demonstrated in an in vivo study (Maresca et al., 2006). Hence, our study confirms that the melanin produced in vitro has comparable protective effects to melanin produced in vivo. This strengthens the hypothesis that a functional epidermal melanin unit is formed in the tissue-engineered epidermal models. These findings are in line with previous studies that showed a connection between the phototype and the production of antioxidant enzymes by keratinocytes after UVB-irradiation (Bessou et al., 1995). However, from a clinical point of view, UV-radiation also triggers skin inflammation caused by radiation-induced damage to epidermal cells. The release of mediators leads to dilation of the vessels and subsequently to the five typical signs of inflamed skin: redness, swelling, heat, burning pain as well as disturbed functions (Soter, 1990). Although the $\mathrm{UV}_{50}$ value simulates damage to the epidermal cells, subsequent erythema or inflammatory reactions cannot be imitated. For more precise assessment of the sun protection classes in vitro, further parameters might be added and the model developed further in order to mimic an inflammatory reaction.

Pigmented epidermal models could be employed to investigate effects of cosmetic and pharmaceutical substances on skin pigmentation, e.g., of self-tanning agents or skin whitener. Moreover, the $\mathrm{UV}_{50}$ test procedure may be applicable to determine the sun protection factor of new cosmetic products. To prevent critical damage to the skin, the duration of direct sun exposure should be limited to a safe dose. The effect of high doses of sunlight was clearly visible in our model when it was challenged with doses of up to $30 \mathrm{~J} / \mathrm{cm}^{2}$. Signs of UV-induced damage in the viable cell layers could be observed for a dose higher than $10 \mathrm{~J} / \mathrm{cm}^{2}$. Interestingly, the appearance of hydropic degeneration in the keratinocytes was diminished at a dose of $30 \mathrm{~J} / \mathrm{cm}^{2}$ but fragmented nuclei still could be observed. These results indicate that doses of 10 to $20 \mathrm{~J} / \mathrm{cm}^{2}$ still allow some cellular reactions, whereas higher doses result in immediate cell death.

Using the $\mathrm{UV}_{50}$ value, we could confirm the protective effect of a commercially available sun lotion. With the sun protective agent, $\mathrm{UV}_{50}$ increased 3-fold from $6.7 \mathrm{~J} / \mathrm{cm}^{2}$ to $21.6 \mathrm{~J} / \mathrm{cm}^{2}$. Interestingly this increase is less than the stated protective effect of a 30 -fold prolonged time of safe sun exposure. So far, the light protection factor is determined according to a standardized human in vivo test method of the European cosmetics association Cosmetics Europe where test persons are exposed to UV-radiation after a standardized application of preparations (Gardiner et al., 2006). However, the safe time determined by this method strongly depends on multiple extrinsic and intrinsic factors. As the interplay of the intrinsic and extrinsic factors is complex, an individual safe sun exposure time cannot easily be assessed. Hence, the method presented here may help to quantify the effects of UV-doses on different skin phototypes in a highly standardized experimental setup and without potential endangerment of test persons by the exposure to UV-radiation. In addition to the SPF analyzed in this study, UVA-PF is an important factor to quantify the protective effect of consumer products. UVB penetrates less deeply into the skin and is for the most part absorbed in the epidermis and superficial dermis. Here, it mainly interacts with keratin, melanin and connective tissue fibers. In contrast, UVA can indirectly damage DNA by inducing free oxygen radicals. It penetrates deeper into the skin and exerts its effect especially in the stratum basale of the epidermis. In further studies, the developed model will be used to determine the UVA-PF and assess damage by either UVA or UVB, which is feasible using the described experimental setup. 


\section{References}

Alonso, L. and Fuchs, E. (2003). Stem cells of the skin epithelium. Proc Natl Acad Sci U S A, Suppl 1, 11830-11835. doi:10.1073/pnas.1734203100

Arakawa, A., Siewert, K., Stohr, J. et al. (2015). Melanocyte antigen triggers autoimmunity in human psoriasis. $J$ Exp Med 212, 2203-2212. doi:10.1084/jem.20151093

Bagozzi, R. P. and Dholakia, U. M. (2006). Open source software user communities: A study of participation in linux user groups. Management Science 52, 1099-1115. doi:10.1287/ mnsc. 1060.0545

Bessou, S., Surlève-Bazeille, J.-E., Sorbier, E. and Taïeb, A. (1995). Ex vivo reconstruction of the epidermis with melanocytes and the influence of UVB. Pigment Cell Res 8, 241-249. doi:10.1111/j.1600-0749.1995.tb00670.x

Brenner, M. and Hearing, V. J. (2008). The protective role of melanin against UV damage in human skin. Photochem Photobiol 84,539-549. doi:10.1111/j.1751-1097.2007.00226.x

Duval, C., Regnier, M. and Schmidt, R. (2001). Distinct melanogenic response of human melanocytes in mono-culture, in co-culture with keratinocytes and in reconstructed epidermis, to UV exposure. Pigment Cell Res 14, 348-355. doi:10.1034/ j.1600-0749.2001.140506.x

Fitzpatrick, T. B. and Breathnach, A. S. (1963). (The epidermal melanin unit system). Dermatol Wochenschr 147, 481-489.

Gardiner, J., Makino, T. and Heerink, B. (2006) International sun protection factor (SPF) test method). COLIPA Guidelines.

Groeber, F., Engelhardt, L., Lange, J. et al. (2016a). A first vascularized skin equivalent for as an alternative to animal experimentation. ALTEX 33, 415-422. doi:10.14573/altex.1604041

Groeber, F., Schober, L., Schmid, F. F. et al. (2016b). Catch-up validation study of an in vitro skin irritation test method based on an open source reconstructed epidermis (phase II). Toxicol In Vitro 36, 254-261. doi:10.1016/j.tiv.2016.07.008

Hertel, G., Niedner, S. and Herrmann, S. (2003). Motivation of software developers in open source projects: An internet-based survey of contributors to the linux kernel. Res Policy 32, 11591177. doi:10.1016/S0048-7333(03)00047-7

Kadekaro, A. L., Kavanagh, R. J., Wakamatsu, K. et al. (2003). Cutaneous photobiology. The melanocyte vs. the sun: Who will win the final round? Pigment Cell Res 16, 434-447. doi:10.1034/j.1600-0749.2003.00088.x

Kaidbey, K. H., Agin, P. P., Sayre, R. M. et al. (1979). Photoprotection by melanin - A comparison of black and caucasian skin. J Am Acad Dermatol 1, 249-260. doi:10.1016/S01909622(79)70018-1

Lakhani, K. R. and von Hippel, E. (2003). How open source software works: "Free" user-to-user assistance. Res Policy 32, 923-943. doi:10.1016/S0048-7333(02)00095-1

Leary, M. R. and Jones, J. L. (1993). The social psychology of tanning and sunscreen use: Self-presentational motives as a predictor of health risk1. J Appl Soc Psychol 23, 1390-1406. doi:10.1111/j.1559-1816.1993.tb01039.x

Liu, Y., Suwa, F., Wang, X. et al. (2007). Reconstruction of a tissue-engineered skin containing melanocytes. Cell Biol Int 31, 985-990. doi:10.1016/j.cellbi.2007.03.009

Maresca, V., Flori, E., Briganti, S. et al. (2006). UVA-induced modification of catalase charge properties in the epidermis is correlated with the skin phototype. J Invest Dermatol 126, 182-190. doi:10.1038/sj.jid.5700021

Mayer, T. C. (1973). The migratory pathway of neural crest cells into the skin of mouse embryos. Dev Biol 34, 39-46. doi:10.1016/0012-1606(73)90337-0

Mewes, K. R., Fischer, A., Zoller, N. N. et al. (2016). Catch-up validation study of an in vitro skin irritation test method based on an open source reconstructed epidermis (phase I). Toxicol In Vitro 36, 238-253. doi:10.1016/j.tiv.2016.07.007

Nordlund, J. J. (2007). The melanocyte and the epidermal melanin unit: An expanded concept. Dermatol Clin 25, 271-281, vii. doi:10.1016/j.det.2007.04.001

Rittié, L. and Fisher, G. J. (2002). UV-light-induced signal cascades and skin aging. Ageing Res Rev 1, 705-720. doi:10.1016/ S1568-1637(02)00024-7

Slominski, A., Moellmann, G., Kuklinska, E. et al. (1988). Positive regulation of melanin pigmentation by two key substrates of the melanogenic pathway, 1-tyrosine and 1-dopa. J Cell Sci 89, 287-296.

Soter, N. A. (1990). Acute effects of ultraviolet radiation on the skin. Semin Dermatol 9, 11-15.

Ullrich, S. E. (2002). Photoimmune suppression and photocarcinogenesis. Front Biosci 7, d684-703. doi:10.2741/A804

Wagner, R. Y., Luciani, F., Cario-Andre, M. et al. (2015). Altered E-cadherin levels and distribution in melanocytes precede clinical manifestations of vitiligo. J Invest Dermatol 135, 1810-1819. doi:10.1038/jid.2015.25

Wang, J. X., Fukunaga-Kalabis, M. and Herlyn, M. (2016). Crosstalk in skin: Melanocytes, keratinocytes, stem cells, and melanoma. J Cell Commun Signal 10, 191-196. doi:10.1007/ s12079-016-0349-3

Wolber, R., Schlenz, K., Wakamatsu, K. et al. (2008). Pigmentation effects of solar-simulated radiation as compared with UVA and UVB radiation. Pigment Cell Melanoma Res 21, 487-491. doi:10.1111/j.1755-148X.2008.00470.x

Yoon, T. J., Lei, T. C., Yamaguchi, Y. et al. (2003). Reconstituted 3 -dimensional human skin of various ethnic origins as an in vitro model for studies of pigmentation. Anal Biochem 318, 260-269. doi:10.1016/S0003-2697(03)00172-6

\section{Conflict of interest}

The authors declare no conflict of interest.

\section{Acknowledgements}

The authors kindly thank the "Fraunhofer Zukunftsstiftung" and the "Bayern Fit Programm" of the Bavarian Ministry of Economic Affairs, Energy and Technology for financially supporting the study. 